\title{
Titel/Title: Die konfessionelle Prägung des deutschen Wohlfahrtsstaats - Vergleich und Bestandsaufnahme
}

Autor*innen/Author(s): Philip Manow

Veröffentlichungsversion/Published version: Postprint

Zeitschriftenartikel/Journal article

\section{Empfohlene Zitierung/Recommended citation:}

Manow, Philip (2018): Die konfessionelle Prägung des deutschen Wohlfahrtsstaats Vergleich und Bestandsaufnahme. In: Sozialer Fortschritt, 67 (6), S. 415 - 431.

Verfügbar unter/Available at:

(wenn vorhanden, bitte den DOI angeben/please provide the DOI if available)

https://doi.org/10.3790/sfo.67.6.415

Zusätzliche Informationen/Additional information:

Der Autor kann kontaktiert werden unter:

manow@uni-bremen.de 


\section{Die konfessionelle Prägung des deutschen Wohlfahrtsstaats - Vergleich und \\ Bestandsaufnahme \\ Philip Manow}

\section{Zusammenfassung}

Der Beitrag entwirft ein Argument zur Rolle religiöser Konflikte in der Entstehung der bekannten Wohlfahrtsstaatsregime Skandinaviens, Kontinentaleuropas, der angelsächsischen Länder und Südeuropas. Er entwickelt auch eine kurze Einordnung eines gemischt-konfessionellen Landes wie Deutschlands in dieses vergleichende Erklärungskonzept. Der Beitrag schließt mit einem Ausblick auf die kürzlichen Sozialstaatsreformen in Deutschland und deren Umgang mit der hergebrachten konfessionellen Prägung des deutschen Wohlfahrtsstaatsmodells.

\section{Abstract: Religious Influences in the German Welfare Regime - Comparison and Survey}

The article develops an argument with respect to the role of religious conflicts in the emergence of different welfare state regimes in Scandinavia, the anglosaxon countries and in continental and southern Europe. It then integrates the German case of a mixed-denominational country into this comparative explanatory framework. The article finally discusses how recent welfare state reforms have dealt with the religious influences that had become manifest in the German welfare regime. JEL-Klassifizierung: I30, I38

\section{Einleitung}

Wenn von der konfessionellen Prägung eines Sozialstaates gesprochen wird, wird oft zunächst an einen manifesten oder latenten Einfluss kirchlicher Soziallehren gedacht (Troeltsch 1923; Kahl 2004; Kahl 2005). Aus der disziplinären Perspektive der Politikwissenschaft ist es hingegen naheliegend, von einer politisch vermittelten konfessionellen Prägung auszugehen, noch spezifischer von einer parteipolitisch vermittelten. Das lenkt die Aufmerksamkeit weg von den $(\leftarrow$ S. 415) kirchlichen Soziallehren, ohne dass man ihren Einfluss bestreiten müsste, und hin zu den gesellschaftlichen Spaltungslinien, die sich parteipolitisch verfestigt haben, ganz konkret hin zu den ,parties of religious defense“ (Stein Rokkan) bzw. den Konsequenzen für den Wohlfahrtsstaat, die aus der Anwesenheit oder Abwesenheit dieses konfessionell begründeten Parteientypus folgen. Ein ausschließlich parteipolitischer Fokus wäre im europäischen Vergleich aber vielleicht auch wiederum zu eng, denn es gibt ja auch Länder mit starkem Staat/ Kirche-Konflikt, wie etwa Spanien oder Frankreich, ohne 
dass sich dieser dort immer auch in entsprechenden Parteibildungen, sprich vor allem in christdemokratischen Parteien, manifestiert hätte (Kalyvas 1996; Kalyvas and Kersbergen 2010) .

Das seit einiger Zeit wiedererwachte Interesse an dem Thema der konfessionellen Prägung von Sozialstaaten begründet sich unter anderen mit der Beobachtung einer offensichtlichen Entsprechung zwischen Typen der Sozialstaatlichkeit und den jeweiligen konfessionellen Verhältnisse eines Landes. So erscheint es als nicht-zufälliger Zusammenhang, dass konservativ-korporatistische Sozialstaaten in Ländern mit Einfluss des Katholizismus zu finden sind, der liberal-angelsächsische Sozialstaat in Ländern mit einem starken Einfluss des reformierten Protestantismus, sozialdemokratisch-skandinavische Wohlfahrtsstaaten hingegen in Ländern mit lutherischer Staatskirche (Gorski 2003, S. 163; Manow 2002). Im Zusammenhang mit einer solchen Beobachtung stellt sich dann jedoch unmittelbar die Frage, wie genau man diese offensichtlichen Übereinstimmungen historisch-genetisch erklären will, oder ob man sie als eine bloße ,konfessionelle Korrelation “ versteht und damit gerade als Indiz für die eher diffuse, vermittelnde Einflussmacht der besonderen ,kulturellen Potenz ' der Religion (F. W. Graf). Das wiederum ließe sich dann sicherlich plausibel begründen, aber nur schlecht empirisch belegen. Für den deutschen Fall ergibt sich darüber hinaus die Notwendigkeit, der mischkonfessionellen Lage Rechnung zu tragen. Wie soll man sich die konfessionellen Einflusswege vorstellen in Ländern, in denen sowohl der Katholizismus als auch der Protestantismus jeweils als große Religionsgruppen vorherrschen?

Ich will in diesem Beitrag zum einen kurz ein Argument skizzieren, das die Korrelation mit einer Kausalität unterlegt, das also - mit etwas weniger Jargon formuliert - erklären kann, wie die jeweiligen konfessionellen Verhältnisse mit den unterschiedlichen Formen der Sozialstaatlichkeit ursächlich zusammenhängen. Ich werde dann genauer auf den komplexeren deutschen Fall und hier insbesondere auf jüngere Entwicklungen eingehen. Eine kurze Zusammenfassung schließt den Beitrag ab. $(\leftarrow$ S. 416)

\section{Das Argument}

In der vergleichenden Wohlfahrtsstaatsliteratur ist es weitgehend unstrittig, mindestens drei verschiedene ,Regime“ entwickelter Sozialstaatlichkeit zu unterscheiden (Esping-Andersen 1990): ein sozialdemokratisch-skandinavisches, ein konservativ-kontinentaleuropäisches und ein liberalangelsächsisches Regime. In der Diskussion über die Gründe, warum sich diese drei Wohlfahrtsstaatsmodelle historisch ausgebildet haben, ist seit Kurzem ein zunächst vielleicht eher überraschender Erklärungskandidat aufgetaucht, und auch einer, der prima facie nichts mit Religion zu tun hat: das Wahlsystem (und darüber vermittelt das Parteiensystem). In aller gebotenen Kürze lautet das Argument, dass Länder mit relativer Mehrheitswahlsystem residuale (liberale) 
Wohlfahrtsstaaten ausbilden, weil sich im Regelfall in den damit vorherrschenden ZweiParteiensystemen keine Umverteilungskoalitionen aus Arbeiterschaft und Mittelschicht formen (Iversen/Soskice 2006). ${ }^{1}$ In den Ländern mit Verhältniswahlrecht bilden sich solche ProWohlfahrtsstaatskoalitionen, aber je nach Parteiensystem, und das heißt, je nach dominanten politischen Spaltungslinien, finden wir (mindestens) zwei unterschiedliche Ausprägungen vor. In den nordischen Ländern mit lutherischer Staatskirche führt die „nationale Revolution“ (Stein Rokkan) nicht zu einem Staat-Kirche-Konflikt, dieser Konflikt findet sich jedoch in den Ländern mit katholischem Bevölkerungsanteil und führt dort meist zur Formierung des politischen Katholizismus in der Gestalt christdemokratischer Parteien. Stattdessen gründen sich in Skandinavien im Zuge der industriellen Revolution auf der Grundlage des Konflikts zwischen Erstem und Zweitem Sektor beziehungsweise zwischen Land und Stadt Agrarparteien, die wir wiederum in den anderen europäischen Parteiensystemen nicht finden - denn dort werden ja die Interessen der frommen Landbevölkerung im Regelfall von christdemokratischen Parteien oder gleich direkt von der katholischen Kirche vertreten. Der Vorschlag lautet nun, den kontinental-konservativen Wohlfahrtsstaat als das Resultat einer teils impliziten, teils expliziten Koalition aus Christ- und Sozialdemokratie zu verstehen, den nordischen Wohlfahrtsstaat hingegen als Resultat einer Koalition aus Sozialdemokratie und Agrarparteien.

Angesichts des in der Literatur sehr häufig zusätzlich identifizierten südlichen Wohlfahrtsstaatstypus stellt sich die Frage, ob er in dieses analytische Modell integriert werden kann (Ferrara 1996; Bonoli 1997; Rhodes 1997; Castles 2005; Ferrera 2010; Gal 2010; León/Guillén 2011). Für Südeuropa ist eine Besonderheit darin zu sehen, dass sich dort neben christdemokratischen Parteien, (૯ S. 417) die aber auch nicht überall zu finden sind, aufgrund der Schärfe des Konflikts zwischen klerikalem und anti-klerikalem Lager stimm- und organisationsstarke kommunistische Parteien ausgebildet haben (und somit auch relativ starke kommunistische Gewerkschaften) (Watson 2015). Die Spaltung der Linken, so zumindest die Hypothese (Manow 2015), hing eng mit der antirepublikanischen Position der katholischen Kirche zusammen - war also in erster Linie eine Erbschaft des Staat-Kirche-Konflikts, der in den monokonfessionell katholischen Ländern Südeuropas besonders vehement ausfiel (Martin 1978).

Wie schlägt sich das nun institutionell und im jeweiligen Leistungsprofil der Wohlfahrtsstaatsmodelle nieder? ${ }^{2}$ In den angelsächsischen Ländern, in denen aufgrund des relativen

\footnotetext{
${ }^{1}$ Durchaus wichtig ist der Unterschied zwischen relativem (USA, Großbritannien) und absolutem Mehrheitswahlrecht (Bsp. Deutsches Kaiserreich, Frankreich III. und V .Republik), weil das absolute Mehrheitswahlrecht zu Mehrparteiensystemen führt.

${ }^{2}$ Das Folgende wird systematischer herausgearbeitet in Manow et al. 2018. Die Darstellung hier muss zwangsläufig knapp und zugespitzt ausfallen und auf das jeweilige langfristige Gleichgewicht abstellen. Sie bezieht sich zudem auf die Phase
} 
Mehrheitswahlrechts konservative Parteien sich häufiger als linke Parteien in der Regierung wiederfinden (Iversen/Soskice 2006; Döring/Manow 2017), bleibt das Ausmaß staatlicher Umverteilung vergleichsweise gering, der Wohlfahrtsstaat ist residual. Die Mittelklasse wird in ihrem Interesse an der Absicherung grundlegender Risiken wie Krankheit, Alter, Arbeitslosigkeit, oder auch bei der Bildung, auf privatwirtschaftliche Lösungen verwiesen (Iversen/Stephens 2008; Ansell/Gingrich 2013). Der Staat zeigt sich nur zuständig als Letztinstanz in Fällen echter Armut, dabei ist die Gewährung von Sozialhilfe an Bedürftigkeitstests gebunden. Die industriellen Beziehungen sind marktdominiert, es gibt keine korporatistische Koordination zwischen Unternehmern und Gewerkschaften, und eine nur schwache Regulierung der Beschäftigungsverhältnisse wie zum Beispiel einen geringen Kündigungsschutz. Es bestehen Entsprechungen zur liberalen Marktökonomie (Hall/Soskice 2001). Im liberalen Typus ist die Lohnungleichheit hoch, diese Länder entwickeln einen substantiellen Niedriglohnsektor (Huber/Stephens 2001), im Übergang zur Dienstleistungsökonomie erlaubt die Lohndifferenzierung substantielles Beschäftigungswachstum im Bereich niedrigproduktiver Dienstleistungen (Wren/Iversen 1998; Wren et al 2013). Marktlösungen herrschen auch bei der Familienpolitik vor: diejenigen, die es sich leisten können, bezahlen private Kinderbetreuung und folgen einem Doppelverdienermodell, für andere gilt aus ökonomischen Gründen weiterhin das Modell des männlichen Alleinverdieners oder die Kombination aus männlicher Voll- und weiblicher Teilbeschäftigung (Lewis et al. 2008; Huber et al. 2009).

Fast alle generösen Wohlfahrtsstaaten finden sich in Ländern mit Verhältniswahl (Ausnahme: Frankreich, hier aber absolutes Mehrheitswahlrecht im Gegensatz zum liberalen Modell), und haben daher Parteiensysteme mit einer höheren $(\leftarrow$ S. 418) ,effektiven Zahl an Parteien“ (Laakso/Taagepera 1979). ${ }^{3}$ Innerhalb dieser Welt der stärker umverteilenden Wohlfahrtsstaaten können wir grundsätzlich drei Regimetypen unterscheiden, und ihnen entsprechen parteipolitische Koalitionsmuster, die die jeweiligen politischen Systeme über die Nachkriegszeit hinweg dominiert haben. In den skandinavischen Ländern herrschten lange Zeit rot-grüne Koalitionen aus Sozialdemokratie und

nach dem Zweiten Weltkrieg.

${ }^{3}$ Die Klassifikation von Frankreich bleibt kontrovers. Zum einen muss man methodisch fragen, ob die Klassifikation von Ländern eigentlich das ist, was man überhaupt theoretisch will oder wollen soll (Shalev 2007). Es sollte, hierin stimme ich mit Michael Shalev vollständig überein, eher darum gehen zu bestimmen, wie weit oder nah eine Politische Ökonomie von einem Typus entfernt ist und ob die Distanz mit der Distanz der politischen Mehrheitsverhältnisse und Koalitionsmuster $\mathrm{zu}$ erklären wäre. Wenn man den großzügigen französischen Wohlfahrtsstaat mit seiner hochentwickelten Familienpolitik betrachtet, dann gehört Frankreich sicherlich zum kontinentalen Typus. Wenn man das Parteiensystem und die Politische Ökonomie generell betrachtet, dann ähnelt Frankreich stärker dem südeuropäischen Typus. Eine starke kommunistische Partei, militante kommunistische Gewerkschaften in einem fragmentierten System der Industriellen Beziehungen, keine Koordinierung und Moderierung der Löhne (Manow/Palier 2009), damit auch kein auf Export basiertes Wachstumsmodell (Iversen/Soskice 2018). Der Staat spielt eine stärkere Rolle in der Wirtschaft, sowohl als Eigentümer, als auch als Nachfrager. Frankreichs dualisierter Arbeitsmarkt ähnelt ebenfalls denen anderer südeuropäischer Länder, insbesondere in Bezug auf die hohe Jugendarbeitslosigkeit. 
Agrarparteien vor, in den kontinentaleuropäischen Ländern hingegen formelle oder informelle Koalitionen aus Christ- und Sozialdemokratie. In den südeuropäischen Ländern schließlich dominierten für lange Zeiträume bürgerliche Sammlungs- und Zentrumparteien wie etwa die Democrazia Cristiana in Italien oder die Gaullisten in Frankreich, vor allem, weil das linke Parteienspektrum in Südeuropa zwischen einer reformistischen Sozialdemokratie und den radikalen Kommunisten gespalten war (Watson 2015). Der zentrale Unterschied zwischen den nordischen und den anderen Ländern mit Verhältniswahlrecht, so die These, war das Fehlen eines starken StaatKirche-Konflikts in Skandinavien, weil hier die lutherische Staatskirche sich nicht nur nicht in einem Konflikt mit dem aufstrebenden Nationalstaat befand, sondern sogar mehr oder weniger identisch mit ihm war (Gustafsson 2003). Stattdessen führte in Nordeuropa der Konflikt zwischen ersten und zweiten Sektor zur Bildung von Agrarparteien (Arter 2001), ein Konflikt, der in den anderen Westeuropäischen Ländern nicht zur Ausbildung eines eigenständigen Parteientypus geführt hat, eben weil hier die Interessen der Landbevölkerung, soweit sie katholisch war, von der Christdemokratie vertreten wurden, oder aber auch direkt von der Kirche.

In Skandinavien kann man den generösen und universalistischen Wohlfahrtsstaat als Manifestation der gemeinsamen Interessen von Bauern- und Arbeiterschaft verstehen, die auch deshalb eine Koalition bilden konnten, weil anders als in Südeuropa kein tiefgreifender sozio-kultureller Konflikt (dort der $(\leftarrow$ S. 419) Konflikt zwischen anti-klerikalen Arbeitern und sehr frommer Landbevölkerung) einer solchen Koalition im Wege stand. So führte das übereinstimmende Interesse der Bauern und Arbeitern an sozialem Aufstieg zu einem inklusiven, einheitlichen Schulsystem, einschließlich einem inklusiven System der höheren Bildung. Dies erwies sich für den Übergang von der Industrie- zur Dienstleistungsökonomie als besonders förderlich, ebenso für die erhöhte Erwerbsbeteiligung der Frauen. So wurde in den sozialdemokratischen Wohlfahrtsstaaten das Doppelverdiener-Familienmodell vorherrschend (Lewis et al. 2008; Hook 2015); was, nebenbei bemerkt, die Frauen der Mittelschicht zu treuen Wählerinnen der Sozialdemokratie machte. Ein umfassendes steuerfinanziertes System der sozialstaatlichen Absicherung, für lange Perioden der Nachkriegszeit staatliche Vollbeschäftigungsgarantien, der korporatistische Austausch zwischen den Spitzenverbänden der Arbeitnehmer und Arbeitgeber unter moderierender Beteiligung der Mittelinks Regierungen - alles dies war die Folge und stabilisierte zugleich die rot-grüne Koalition, die in den skandinavischen Ländern erfolgreich den Strukturwandel von einer „ländlichen Ökonomie zu einer Mittelschichten-Gesellschaft“ bewältigte (Esping-Andersen 1990, S . 32).

Im Gegensatz hierzu herrschten in Kontinentaleuropa entweder formelle oder informelle Koalitionen zwischen Christ- und Sozialdemokratie vor, wobei sozialdemokratische Parteien oftmals Juniorpartner waren und generell nicht so häufig an der Regierung beteiligt waren 
(Döring/Schwander 2015; Döring/Manow 2017), weswegen man die christdemokratischen Parteien als zentral für die Entstehung des kontinentalen Wohlfahrtsstaats bezeichnen kann (Kersbergen 1995; Huber/Stephens 2001; Manow 2004; Kalyvas/Kersbergen 2010). Dieser - und das entsprechende Bildungssystem - spiegeln daher auch die interne Kompromissbildung wider, die Parteien der religious defense bewerkstelligen müssen, da sie sozio-ökonomisch heterogene Gruppen mobilisieren. Deswegen ist der kontinentale Sozialstaatstypus charakterisiert durch begrenzte Umverteilung zwischen verschiedenen Arbeitsmarktgruppen aufgrund des fragmentierten, ,okkupationalistischen“ Charakter der Sozialversicherungssysteme, und durch die Beitragsfinanzierung ist auch die intertemporale Umverteilung innerhalb spezifischer Versichertengruppen (Bauern, Beamte, Angestellte, Arbeiter) weitaus bedeutender als die zwischen verschiedenen Beschäftigten- und damit Risikogruppen . Das führt dazu, dass der Wohlfahrtsstaat insgesamt eher transfer- als service-intensiv ist, damit zeigt er sich zugleich als weniger geeignet für den Übergang von der Industrie- zur Dienstleistungsökonomie, etwa weil einer Ausweitung des öffentlichen (sozialen) Dienstleistungssektors Grenzen gesetzt sind (Wren 2013) . Beitragsfinanzierung und die einkommensabhängige Höhe der Sozialleistungen bieten Prämien auf den Erwerb von spezifischen Berufsqualifikationen und bilden damit auch die Grundlage für das Entstehen kooperativer Beziehungen zwischen Arbeitnehmern und Arbeitgebern, stützen also die koordinierte Form der Marktwirtschaft, die in den $(\leftarrow$ S. 420) kontinentaleuropäischen Ländern vorherrscht. Mit dem Übergang in die Dienstleistungsökonomie verstärkt das allerdings auch die dualisierenden Tendenzen auf den Arbeitsmärkten (Palier/Thelen 2010; Iversen/Soskice 2011).

Das Schulsystem reflektierte für lange Zeit in ganz ähnlicher Weise die heterogenen Interessen zwischen Unter-, Mittel- und Oberschicht. Das gegliederte Schulsystem mit Haupt- und Realschule sowie Gymnasium führte die jeweiligen Schülerkohorten sehr frühzeitig auf verschiedene Karrierepfade - der Hauptschulabschluss qualifizierte für eine Zweitqualifikation im stark entwickelten System der dualen Berufsbildung, die Realschule bereitete für einen Angestelltenberuf vor, das Gymnasium produzierte die im Vergleich relativ kleine Kohorte eines jeden Jahrgangs, die ein Studium anschloss und somit die ,akademisierten` Berufe wie Anwälte oder Ärzte wählen konnte (Ansell/Gingrich 2013). Einer erhöhten Frauenerwerbsbeteiligung standen und stehen oftmals weiterhin ungünstige Steuerregelungen wie das Ehegattensplitting, ein geringer Ausbau der Dienstleistungsbeschäftigung und eine vergleichsweise großzügige, aber auf passive Transfers ausgerichtete Familienpolitik entgegen (Esping-Andersen 1999; Huber/Stephens 2001). Seit den 1990er-Jahren entwickelt sich je- doch das einstmalig dominierende Modell des männlichen Haupterwerbers tendenziell in Richtung auf ein familiäres Eineinhalb-Erwerbsmodell, das teilweise auch bereits in die skandinavische Richtung der Doppelverdienerfamilie weist (Lewis 2001; Lewis et 
al. 2008; Pettit/Hook 2009).

Wenn wir wieder das Parteiensystem als unseren analytischen Ausgangspunkt nehmen, so ist die differentia specifica der südeuropäischen Länder die Spaltung des linken Parteienspektrums in einen reformistischen, sozialdemokratischen und einen radikalen, kommunistischen Flügel (für das Folgende Manow 2015). Diese Spaltung zeichnete lange Zeit für die Dominanz rechtsbürgerlicher Parteien oder Koalitionen verantwortlich (Iversen/Soskice 2015). Die institutionellen Konsequenzen dieser parteipolitischen Konstellation lassen sich wie folgt skizzieren: Die südeuropäische Politische Ökonomie kennt sehr hohe Standards des Beschäftigtenschutzes für einen zumeist männlichen Kern der Beschäftigten, die von militanten und fragmentierten Gewerkschaften gegen alle Reformversuche vehement verteidigt werden. Der inflexible Arbeitsmarkt führt zu hoher Dualisierung, insbesondere aber zu niedriger Frauenbeschäftigung und hoher Jugendarbeitslosigkeit. Für die Jüngeren bedeutet das eine lange Abhängigkeit vom elterlichen Haushalt - die Familie hat generell für vielfältige Lücken in den sozialen Sicherungssystemen des Südens zu kompensieren, sowohl bei den Transfers als auch bei den sozialen Dienstleistungen.

Die starke Arbeitsmarktdualisierung ist in Verbindung mit einem fragmentierten Sozialversicherungsstaat verantwortlich für die starke Ungleichheit zwischen teils deutlich überversorgten Insidern und deutlich unterversorgten Outsidern (Ferrera 2010 .). Auch in den südeuropäischen Politischen Ökonomie ( $\leftarrow$ S. 421) fehlen korporatistische Elemente (Siaroff 1999), aber hier nicht wie in den liberalen Ländern wegen des Vorherrschens deregulierter Arbeitsmärkte, sondern weil die fragmentierten Gewerkschaften miteinander um Mitglieder konkurrieren und sich deswegen in Lohnverhandlungen wechselseitig $\mathrm{zu}$ überbieten trachten, während es auf der Regierungsseite an gemäßigten sozialdemokratischen Parteien mangelt, die gewerkschaftliche Lohnzurückhaltung durch glaubwürdige Sozial- oder Beschäftigungsversprechen motivieren und unterstützen könnten. Die mangelnde Lohnkoordinierung und -moderierung führt zu hoher Inflation und geringer internationaler Wettbewerbsfähigkeit. Regierungen reagierten lange Zeit mit protektionistischen Maßnahmen und/oder periodischen Währungsabwertungen, Firmen mit vergleichsweise niedrigen Investitionen. Im Resultat entwickelte sich in Südeuropa daher kein auf Export basiertes Wachstumsmodell so wie in Kontinentaleuropa, sondern ein Modell, das vornehmlich auf Binnennachfrage setzte (Eichengreen 1996, 2007, insbesondere S. 90, 104 und $114 / 115)$.

Der südeuropäische Sozialstaat ist zudem charakterisiert durch hohe Rentenausgaben (old age welfare state) und - im Gegensatz zum kontinentalen System - nationale Gesundheitsdienste (Ferrera 1996). Einige halten das hohe Ausmaß an Klientelismus und Patronage und generell die geringe 
administrative Kapazität des südlichen Wohlfahrtsstaats für ein weiteres seiner Merkmale (Rhodes 1997, S. 6, 16). Zu diesem Bild gehört eine umfassende Schattenökonomie, auch von Migranten geprägt, sowie ein überproportionaler Anteil an Selbstständigen und Freiberuflern als Beschäftigtengruppen, bei denen bürgerliche Parteien lange Zeit dafür sorgten, dass dieser wichtigen Klientel eine Besteuerung erspart blieb.

In Kontinentaleuropa subventioniert der Staat die Hausfrauenfamilie mit ,passiven“ Leistungen wie Transfers und Steuererleichterungen, während er im Süden die Familie weder unterstützt noch ersetzt. Diese markante women unfriendliness des südlichen Regimes ist nicht zuletzt dem Umstand geschuldet, dass der Parteienwettbewerb um die Stimmen der weiblichen Wählerschaft für lange Zeit verzerrt war (Ignazi/Wellhofer 2013; Morgan 2013; Emmenegger/Manow 2014). Unter anderem erhielten Frauen das Wahlrecht hier vergleichsweise spät (Siaroff 1994, S. 96 - 98), weil sie - so der nicht völlig unbegründete Verdacht der politischen Linken - aufgrund ihrer stärker religiösen Prägung konservativer wählen. Auch das zunächst widersprüchlich erscheinende Nebeneinander eines starken Familialismus, also der starken Rolle der Familie als soziales Unterstützungsnetzwerk, was zunächst sehr ,katholisch“ anmutet, und zugleich der unvergleichlich starken Rolle des Staates im Bildungsbereich, der hier die Familie als Sozialisierungsinstanz weitestgehend verdrängt hat, was nun wieder ganz, unkatholisch“ erscheint, erklärt sich historisch aus den vehementen Schulstreit zwischen Staat und Kirche, ist also religiös bedingt (Morgan 2002, 2006). ( $\leftarrow$ S. 422)

Alles dies zeigt meines Erachtens, dass ein simpler Verweis auf ,Familialismus` und katholischpatriarchalischen Traditionalismus nicht ausreicht, um das südliche Regime zu erklären (aber EspingAndersen 1999, S. 90). Maurizio Ferrera hat - neben anderen - darauf verwiesen, wie sehr die Ungleichgewichtigkeit des südlichen Sozialmodells als das Resultat eines ,specific pattern of political competition“ (Ferrera 2010, S. 622) bewertet werden muss (Watson 2015). So stand etwa die Spaltung der Linken den Nachkriegsreformbemühungen zur Überwindung des hohen berufsspezifischen Zersplitterungsgrads der verschiedenen Sozialversicherungsprogramme entgegen (Ferrera 1996, S. 31), während universalistische Programme dort mehrheitsfähig wurden, wo keine etablierten Privilegien gefährdet waren, etwa in der Gesundheitsversorgung. Eine ganz unmittelbare Konsequenz der Spaltung der Linken war, dass sie seltener Regierungsverantwortung trug. So war beispielsweise die Democrazia Cristiana aufgrund der Abwesenheit eines ernsthaften politischen Konkurrenten bis zum mani pulite-Skandal die hegemoniale Kraft im italienischen Parteiensystem. Wenn sie dann doch einmal an die Regierung gekommen war, konnte die Linke oft nicht ihre Reformziele erreichen, weil sie sich mit der Konkurrenz einer immer radikaler positionierten Parteialternative konfrontiert sah (Hopkin 2004). 
Aber wie passt nun ein gemischt-konfessionelles Land wie Deutschland in dieses Bild? Dieser Frage ist der folgende Abschnitt gewidmet.

\section{Der deutsche Sozialstaat in komparativer Perspektive}

Vor dem Hintergrund des oben skizzierten Arguments hinsichtlich der Entwicklung der vier Wohlfahrtsstaatsregime stellt sich die spezifischere Frage nach der Einordnung des deutschen Falls. Hinsichtlich seiner religiösen Prägung haben die Forschungen von Florian Tennstedt und Christoph Sachße, von Hans-Günter Hockerts, von Franz-Xaver Kaufmann, Karl Gabriel und anderen bereits reichhaltige Erkenntnisse erbracht. Deswegen braucht es hier auch nur um eine genauere Einordnung des bundesdeutschen Falls in den skizzierten Vergleichsrahmen zu gehen. Die ist in der Literatur bislang kaum entwickelt worden. Ich skizziere erneut das Argument nur in seinen Grundzügen (Manow 2001, 2008).

Die ursprünglich protestantischen Wurzeln der Sozialreform des Kaiserreichs sind bekannt. Sie war ein Modernisierungsprojekt von oben, getragen von Bürokratie und Bildungselite und somit automatisch ein protestantisches Unterfangen. Es war ein durchaus interventionsfreundliches, etatistisches Projekt des , socialen Kaisertums'. Die weitere historische Entwicklung ist nun allerdings ironisch. Elemente der Selbstverwaltung, ursprünglich mit disziplinierender Absicht in die Sozialversicherungsgesetzgebung hineingeschrieben, eröffneten Möglichkeiten der Integration von sowohl sozialistischer als auch katholischer $(\leftarrow$ S. 423) Arbeiterschaft in den Wohlfahrtsstaat und dadurch auch in das politische System des Kaiserreichs (Manow 1997). Der Wohlfahrtsstaat wurde $\mathrm{zu}$ einer zentralen organisatorischen und finanziellen Ressource der Gewerkschaften. Sozialdemokratie und die Bewegung des politischen Katholizismus ,kaperten“ somit das ursprünglich protestantisch-obrigkeitsstaatliche Projekt. Der Wohlfahrtsstaat bot mit seinen korporatistischen Strukturen diesen zwei ,Reichsfeinden“ Möglichkeiten zur Integration in das Wilhelminische Deutschland in Form einer ,negativen Integration“ (Dieter Groh), also als Lager, und legte damit die Voraussetzung für deren dominante Stellung in der Zwischenkriegszeit (Sachße/Tennstedt 1988).

Im protestantisch-bürgerlichen Lager provozierte diese Entwicklung eine zunehmende Entfremdung und Abwendung vom einstigen Lieblingsprojekt, der Sozialreform. Denn mit dessen Kaperung durch Sozialdemokratie und politischen Katholizismus sah sich nun ein tiefgreifend „statusverunsichertes“ Bürgertum zunehmend wirtschaftlich aufgerieben zwischen den organisierten Großgruppen von Arbeit und Kapital und ihrem ,inflationären Konsens`, empfand sich als politisch 
marginalisiert, ohne Repräsentation durch eine macht- volle Partei, fühlte sich aber schließlich auch konfessionell in die Enge getrieben. Die bürgerlich-protestantische Bildungselite empfand den Weimarer Korporatismus als handfeste ökonomische Bedrohung und zugleich als Herausforderung eines konfessionellen Dominanzanspruchs, den der lutherische Protestantismus seit 1871 immer für sich beansprucht hatte. Das führte zur Abwendung vom eigenen sozialstaatlichen Projekt, denn von dem kräftig expandierenden Wohlfahrtsstaat schienen vor allem die christlichen (katholischen) und freien (sozialistischen) Gewerkschaften, entsprechend parteipolitisch Zentrum und Sozialdemokratie zu profitieren Die Gewerkschaften waren gut in die Selbstverwaltung des Bismarck'schen Sozialstaates integriert, während der soziale Protestantismus ein heterogenes, aber bei aller Heterogenität doch durchgängig (bildungs-)bürgerliches Projekt blieb. Ohne Mobilisierungserfolg in den Arbeiterschichten fehlte ihm die organisatorische Verbindung mit dem besonders auf deren Interessen ausgerichteten deutschen Wohlfahrtsstaat. Nur in der Fürsorge, ein eher nachrangiges Betätigungsfeld im deutschen Sozialversicherungsstaat, auf die zudem in der Weltwirtschaftskrise zunehmend alle sozialpolitische Anpassungslast abgeladen wurde, konnte der soziale Protestantismus ein genuines Betätigungsfeld finden (vom Bruch 1985). Das bürgerliche (protestantische) Lager schwenkte daher in das Lager der Wohlfahrtsstaatskritik. Diese Kritik mündete dann insbesondere in eine Wirtschaftsdoktrin, die sozialen Frieden und Ausgleich nicht mehr vom Wohlfahrtsstaat erwartete, sondern auf Wirtschaftspolitik, genauer Ordnungspolitik setzte. Die beste Sozialpolitik sei eine erfolgreiche Produktionspolitik, so lautetet die neue Formel, ursprünglich bei Heinrich Herkner, dem Vorsitzenden des Vereins für Socialpolitik, später dann wortgleich bei Ludwig Erhard und Walter Eucken. $(\leftarrow$ S. 424)

Wenn man aus dieser Perspektive auf die Gründungsphase der Bundesrepublik blickt, lässt sich durchaus sagen, dass das gemischt-konfessionelle Deutschland nach 1945 in Fragen seiner Politischen Ökonomie auch einen zwischen-konfessionellen Kompromiss gefunden hat, auch wenn sich dieser eher institutionell einstellte als bewusst geschlossen wurde (Manow 2000, 2018). Zum einen lebte die ,Krypto-Groß-Koalition“ von Sozialdemokratie und Zentrum in allen Fragen der Sozialpolitik weiter, eigentlich bis 1996, als mit einer Rentenreform in der Spätphase der Kohljahre zum ersten Mal in der Geschichte der Bundesrepublik ein größeres Sozialgesetz nicht auf die gemeinsame Zustimmung von CDU/CSU und SPD traf. Darüber hinaus beruhte das deutsche Wirtschaftsmodell in der Nachkriegszeit aber auf einem institutionalisierten Kompromiss zwischen einem Pro-Wohlfahrtsstaats-Lager aus Arbeiterbewegung und Sozialkatholizismus einerseits und einem wohlfahrtsstaatsskeptischen ordoliberalen Lager des protestantischen Bürgertums andererseits. Den Kompromiss zwischen liberaler Wirtschaftspolitik und ,katholischer Sozialpolitik‘ verkörperte die neue CDU als ,Union der Bekenntnisse“ besonders gut. Hierfür stand die Christlich- 
Demokratische Arbeitnehmerschaft CDA auf der einen Seite, und der evangelische Arbeitskreis innerhalb der CDU, in dem exponierte Ordoliberale wie Alfred Müller-Armack oder Franz Böhm aktiv waren, auf der anderen. Die institutionelle Sphärentrennung zwischen Wirtschafts- und Sozialpolitik, zwischen den Bundesministerien für Wirtschaft und Arbeit, und vor allem auch zwischen steuerfinanziertem Staatshaushalt und beitragsfinanziertem Sozialhaushalt, bedeutete aber auch, dass der Expansion des Wohlfahrtsstaats zunächst kaum Hindernisse in den Weg gelegt wurden. Die Ordoliberalen hatten ihre Wirtschaftsdomäne, der Sozialkatholiken ihre sozialpolitische Domäne. Spätestens nach der Rentenreform von 1957 war diese Teilung etabliert (Abelshauser 1996). So kann man sicherlich sagen, dass der deutsche Wohlfahrtsstaat - und wohl auch die deutsche Politische Ökonomie generell -,,um zwei Spannungslinien der deutschen Gesellschaft herum gebaut“ wurde: „die Klassenspannung wie die konfessionelle Spannung“ (Gabriel/Reuter 2013, S. 103). Was sind die Langfristkonsequenzen dieser Interaktion von Klasse und Konfession in der bundesdeutschen Frühphase? Gibt es noch heute messbare Gravitationswellen ihres ursprünglichen Zusammenstoßes? Oder haben sich diese Konflikte schlicht überlebt? Ich will diese Frage im Schlussabschnitt knapp versuchen zu beantworten.

\section{Konfessionelle Gravitationswellen?}

Im Zeitverlauf hat sich in der Bundesrepublik die Virulenz des Konfessionskonflikts ohne jeden Zweifel nachhaltig abgeschwächt, so wie ja generell religiöse Bindungen abgenommen haben. Der mit der Deutschen Einigung verbundene massive Säkularisierungsschub tat dann ein Weiteres. Sozialpolitisch kann $(\leftarrow \mathbf{S . ~ 4 2 5})$ man daher auch eher das Räumen vorheriger Positionen konstatieren als lang anhaltende konfessionelle Kontinuitäten. Die CDU hat insbesondere seit der Jahrtausendwende eine tiefgreifende Modernisierung ihre Familienpolitik vollzogen (vgl. den Beitrag von Agnes Blome in diesem Heft). In der Frage der Ehe für alle, der Mütterrente, der Gleichstellungspolitik et cetera mag es noch Nachhutgefechte eines wertkonservativen Strangs in der CDU und insbesondere CSU geben, aber die Erschließung neuer Wählerschichten hatte unter Merkel erkennbar Vorrang vor der Bewahrung programmatischer Traditionsbestände . Insofern spricht zunächst wenig von einer auch heute noch wichtigen konfessionellen Prägung des sozialpolitischen Geschehens. In dieses Bild passt der - insgesamt misslungene - Versuch einer sozialpolitischen NeuAusrichtung der CDU während des Leipziger Parteitages 2003, der ebenfalls für eine sehr weitreichende, (neo-)liberale Abkehr von sozialpolitischen Grundprinzipien des Bismarck'schen Sozialstaatsmodells stand, etwa mit dem Vorhaben, die Finanzierung der gesetzlichen Krankenversicherung auf Kopfpauschalen umzustellen. Eine besondere Verpflichtungskraft scheinen 
die Sozialversicherungsprinzipien der alten Bundesrepublik in der Merkel-CDU nicht mehr zu besitzen - und auch das erklärt die latenten, teils manifesten Spannungen zwischen CDU und CSU.

Die Agenda 2010 steht ohne Zweifel für die tiefgreifendste Reform des deutschen Sozialstaatsmodells der jüngsten Vergangenheit. Solidarrente und Mindestlohn sind demgegenüber vornehmlich als nachträgliche Teilkorrekturen zu verstehen, die vor allem auf die elektoralen Folgen der Agenda, insbesondere den starken Stimmenverlust der SPD, reagierten (Schwander and Manow 2017). Und auch hier wird man wohl eine Überwindung bisheriger - indirekt konfessioneller Prägungen konstatieren müssen, und nicht deren langes Nachwirken. Denn die Agenda 2010 steht zentral für die Aufkündigung eines zentralen ,Bismarck’schen Versprechens', der Statussicherung, das über die gesamte Nachkriegszeit bis 2005, also recht exakt 60 Jahre, prägend für die deutsche Sozialstaatlichkeit gewesen war (Manow et al . 2018), wobei das kein im engeren Sinne ,konfessionell hergeleitetes‘ Prinzip war, sich aber als besonders hilfreich im Interessenabgleich zwischen unterschiedlichen Berufsgruppen erwiesen hat. Die wichtige ökonomische Funktion, die das Prinzip der Statussicherung in der Vergangenheit für das deutsche Produktionsmodell entfaltet hatte, ist daher auch Ausdruck eben jener parteipolitischen Vermittlungslogik, die intertemporale Umverteilung und vor allem Umverteilung innerhalb bestimmter Berufsgruppen gegenüber Umverteilung zwischen ihnen prämiert hatte. Etwas konkreter gesprochen: für eine Partei wie die $\mathrm{CDU} / \mathrm{CSU}$, der es immer darum gehen musste, eine sozio-ökonomisch diverse Wählerschaft zu repräsentieren und zufrieden $\mathrm{zu}$ stellen, war die Beitragsfinanzierung und die damit quasiautomatisch implizierte Leistungsdifferenzierung nach Beitragshöhe und Beitragsdauer für lange Zeit ein wichtiges Instrument interner Kompromissbildung. ( $\leftarrow$ S. 426)

Doch dieses Prinzip wurde mit der Agenda-Reform an zentraler Stelle, nämlich im vierten Teil der Hartz-Reformen, durch die massive Änderung der maximalen Bezugsdauer des Arbeitslosengeldes von 32 auf 12 Monate und der deutlichen Verschärfung der Zumutbarkeitskriterien unter der Schröder-Regierung aufgekündigt. Auch als die Christdemokraten zurück an der Macht waren, wurden diese Maßnahmen nicht wieder rückgängig gemacht, so wie die CDU/CSU im Gesetzgebungsprozess selber ja auch durchgehend versucht hatte, ihre oppositionelle Bundesratsmehrheit für Reformverschärfungen zu nutzen. Es gibt Hinweise darauf, dass die Neuregelungen des ALG II (wie auch dann 2007 die Heraufsetzung des Renteneintrittsalters) insbesondere in Verbindung mit der ab Herbst 2015 einsetzenden Flüchtlingskrise dazu beigetragen haben, dass gerade unter den Arbeitern der rechtspopulistische Protest um sich griff (Manow/Schwander 2018). Insofern stünde die weitreichende Reform der Arbeitslosenversicherung unter dem zweiten Schröder-Kabinett im engen Zusammenhang mit der deutlichen Fragmentierung 
des deutschen Parteiensystems, mit zunächst der nachhaltigen bundesweiten Etablierung der PDS/die Linke, dann mit der weiteren Zersplitterung im rechten Lager.

Es gibt gute Gründe, die Agenda-Reformen angesichts der sehr hohen Arbeitslosigkeit 2002 - 2005 für notwendig und insgesamt auch erfolgreich zu halten. Ob man nun aber zu einer solchen Einschätzung oder zu einer kritischeren Position gelangt, davon unbenommen ist die Feststellung, dass die Agenda 2010 zusammen mit anderen Entwicklungen - hier insbesondere der Transferierung der Geldpolitik auf die europäischen Ebene - für ein Ende des polit-ökonomischen Nachkriegskonsenses der Bundesrepublik steht, und damit auch für eine Abkehr von den in ihm eingekapselten konfessionellen Einflüssen.

\section{Literatur}

Abelshauser, W. (1996): Erhard oder Bismarck? Die Richtungsentscheidung der deutschen Sozialpolitik am Beispiel der Reform der Sozialversicherung in den Fünfziger Jahren, in: Geschichte und Gesellschaft 22, S. 376 - 392.

Ansell, B./Gingrich, J. (2013): A Tale of Two Dilemmas: Varieties of Higher Education and the Service Economy, in: Wren, A. (Hrsg.), The Political Economy of the Service Transition. Oxford, S. 195 224.

Arter, D. (2001): From Farmyard to City Square? The Electoral Adaption of the Nordic Agrarian Parties, Aldershot.

Bonoli, G. (1997): Classifying Welfare States: A Two-Dimension Approach, in: Journal of Social Policy 26,3, S. $351-372$.

Bruch, R. vom (1985): Bürgerliche Sozialreform im deutschen Kaiserreich, in: ders., Weder Kommunismus noch Kapitalismus. Bürgerliche Sozialreform in Deutschland vom Vormärz bis zur Ära Adenauer. München, S. $61-179 .(\leftarrow$ S. 427)

Castles, F. G. (2005): The Welfare State and Democracy: On the Development of Social Security in Southern Europe, 1960 - 1990, in: Gunther, R et al. (Hrsg.), Democracy and the State in the New Southern Europe . Oxford, S. $42-86$.

Döring, H./Manow, P. (2017): Is PR More Favourable to the Left? Electoral Rules and Their Impact on Elections, Parliaments and Cabinet Formation, in: British Journal of Political Science 47,1, S. 149 -164 . 
Döring, H./Schwander, H (2015): Revisiting the Left Cabinet Share: How to Measure the Partisan Profile of Governments in Welfare State Research, in: Journal of European Social Policy 25,2, S. $175-193$.

Eichengreen, B. (1996): Institutions and economic growth: Europe after World War II, in: Crafts, N./Toniolo, G. (Hrsg.), Economic Growth in Europe since 1945. Cambridge, S. 38 - 72.

- (2007): The European Economy since 1945: Coordinated Capitalism and beyond, Princeton.

Emmenegger, P./Manow, P. (2014): Religion and the Gender Vote Gap: Women's Changed Political Preferences from the 1970s to 2010, in: Politics \& Society 42,2, S. 166 - 193.

Esping-Andersen, G. (1990): The Three Worlds of Welfare Capitalism, Cambridge.

- (1999): The Social Foundation of Postindustrial Economies, Princeton.

Ferrera, M. (1996): The Southern Model of Welfare in Social Europe, in: Journal of European Social Policy 6,1, S. $17-37$.

- (2010): The South European Countries in: Castles, F. G. et al.(Hrsg.), The Oxford Handbook of the Welfare State, Oxford, S. $616-629$.

Gabriel, K./Reuter, H.-R. (2013): Religion und Wohlfahrtsstaatlichkeit in Deutschland. Korporatistischer Sozialversicherungsstaat und konfessionelle Prägung, in: dies. u. a. (Hrsg.), Religion und Wohlfahrtsstaatlichkeit in Europa, Tübingen, S. 93 - 140.

Gal, J. (2010): Is there an Extended Family of Mediterranean Welfare States?, in: Journal of European Social Policy 20, S. $283-300$.

Gustafsson, G. (2003): Church-State Separation Swedish Style, in: Madeley, J. T. S./Enyedi, Z. (Hrsg.), Church and State in Contemporary Europe. The Chimera of Neutrality. Special Issue of West European Politics 26,1, S. $51-72$.

Hall, P. A./Soskice, D. (Hrsg.), Varieties of Capitalism. The Institutional Foundations of Comparative Advantage, Oxford.

Hook, J. L. (2015): Incorporating, Class' into Work-Family Arrangements: Insights from and for Three Worlds, in: Journal of European Social Policy 25,1, S. 14 - 31.

Hopkin, J. (2004): „Hard Choices, Mixed Incentives: Globalization, Structural Reform, and the 
Double Dilemma of European Socialist Parties.“ Mimeo London School of Economics.

Huber, E. J. et al. (2009): The Politics of Women's Economic Independence, in: Social Politics: International Studies in Gender, State \& Society 16,1, S. 1 - 39.

Huber, E./Stephens, J. D. (2001): Development and Crisis of the Welfare State. Parties and Policies in Global Markets, Chicago. $(\leftarrow$ S. 428)

Ignazi, P./Wellhofer, E. S. (2013): Votes and Votive Candles: Modernization, Secularization, Vatican II, and the Decline of Religious Voting in Italy: 1953 - 1992, in: Comparative Political Studies 46,1, S. $31-62$.

Iversen, T./Soskice, D. (2006): Electoral Institutions and the Politics of Coalitions: Why Some Democracies Redistribute More Than Others, in: American Political Science Review 100,2, S. 165 $-181$.

- (2011): Dualism and Political Coalitions: Inclusionary versus Exclusionary Reforms in an Age of Rising Inequality. Paper Prepared for Presentation at the Annual Meeting of the American Political Science Association, Toronto, S. 3 - 6.

- (2015): Democratic Limits to Redistribution. Inclusionary versus Exclusionary Coalitions in the Knowledge Economy, in: World Politics 67,2, S. 185 - 225.

- (2018): A structural-institutional explanation of the Eurozone crisis, in: Manow, P. et al., Welfare Democracies and Electoral Politics. Oxford.

Iversen, T./Stephens, J. D. (2008): Partisan Politics, the welfare state, and three worlds of human capital formation, in: Comparative Political Studies 41,5, S. 600 - 637.

Kahl, S. (2004): The History of poor relief in Catholic, Lutheran and reformed Protestant countries: some observations and hypotheses, in: Kersbergen, K. van/Manow, P. (Hrsg.), Religion, Class Coalitions and the Welfare State, Cambridge.

- (2005): The Religious Roots of Modern Poverty Policy: Catholic, Lutherian, and Reformed Protestant Traditions Compared in: European Journal of Sociology 46.1, S. 91 - 126.

Kalyvas, S. N. (1996): The Rise of Christian Democracy in Europe, Ithaca.

Kalyvas, S. N./Kersbergen, K. van (2010): Christian Democracy, in: Annual Review of Political Science 13,1, S . $183-209$. 
Kersbergen, K. van (1995): Social Capitalism. A Study of Christian Democracy and the Welfare State, London/New York.

Kersbergen, K. van/Manow, P. (Hrsg.) (2009): Religion, Class Coalitions and the Welfare State. Cambridge Series on Social Theory, Politics and Religion, New York.

Laakso, M./Taagepera, R. (1979): Effective Number of Political Parties. A Measure with Application to West Europe, in: Comparative Political Studies 12,1, S. 3 - 27.

León, M./Guillén, A. M. (2011): The Spanish Welfare State in European Context, Farnham (Surrey).

Lewis, J. (2001): The Decline of the Male Breadwinner Model: Implications for Work and Care, in: Social Politics: International Studies in Gender, State \& Society 8,2, S. 152 - 169.

Lewis, J. et al. (2008): Patterns of Development in Work/Family Reconciliation Policies for Parents in France, Germany, the Netherlands, and the UK in the 2000s, in: Social Politics: International Studies in Gender, State \& Society 15,3, S. $261-286$.

Manow, P. (1997): Social Insurance and the German Political Economy, in: MPIfG Discussion Paper 97,2.

- (2000): „Modell Deutschland“ as an Interdenominational Compromise, Cambridge, CES Working Paper. $(\leftarrow$ S. 429)

- (2001): Ordoliberalismus als ökonomisches Ordnungstheorie, in: Leviathan 29, S. 179 - 198.

- (2002): „The Good, The Bad and the Ugly“، Esping-Andersens Sozialstaats-Typologie und die konfessionellen Wurzeln des westlichen Wohlfahrtsstaats, in: Kölner Zeitschrift für Soziologie und Sozialpsychologie 54,2, S. $203-225$.

- (2004): „The Good, the Bad, and the Ugly“ - Esping-Anderse's Regime Typology and the Religious Roots of the Western Welfare State, in: MPIfG Working Paper 3/04.

- (2008): Religion und Sozialstaat. Die konfessionellen Grundlagen europäischer Wohlfahrtsstaatsregime. Frankfurt am Main.

- (2013): Die religiöse Konfliktlinie, die Spaltung der Linken und die Politische Ökonomie Südeuropas, in: Leviathan 4,41, S. $541-573$.

- (2015): Workers, Farmers, and Catholicism: A History of Political Class Coalitions and the South- 
European Welfare State Regime, in: Journal of European Social Policy 25,1, S. 32 - 49.

- (im Erscheinen): Die religiöse Prägung des bundesdeutschen Wohlfahrtsstaates im europäischen Vergleich, in: Gerster, D./Melis, V. van/Willems, U. (Hrsg.), Religionspolitik heute. Problemfelder und Perspektiven in Deutschland, Freiburg.

- (im Erscheinen, a): Social Protection, Capitalist Production. The Bismarckian Welfare State in the German Political Economy, 1880 - 2015.

Manow, P./Palier, B. (2009): A Conservative Welfare State Regime without Christian Democracy? The French État-Providence, 1880 - 1960. Religion, in: Kersbergen, K. van/Manow, P. (Hrsg.), ClassCoalitions and the Welfare State, New York, S. 146 - 175.

Manow, P. et al. (Hrsg.) (2018): Welfare democracies and party politics: Explaining electoral dynamics in times of changing welfare capitalism, Oxford.

Martin, D. (1978): A General Theory of Secularization, Oxford.

Morgan, K. J. (2002): Forging the Frontiers between State, Church, and Family: Religious Cleavages and the Origins of Early Childhood Education and Care Policies in France, Sweden, and Germany, in: Politics \& Society 30,1, S. $113-148$.

- (2006): Working Mothers and the Welfare State. Religion and the Politics of Work- Family Policies in Western Europe and the United States, Stanford.

- (2013): Path Shifting of the Welfare State: Electoral Competition and the Expansion of WorkFamily Policies in Western Europe, in: World Politics 65,1, S. 73 - 115.

Palier, B./Thelen, K. A. (2010): Institutionalizing Dualism: Complementaries and Change in France and Germany, in: Politics \& Societies 38,1, S. 119 - 148.

Pettit, B./Hook, J. L. (2009): Gendered Tradeoffs: Family, Social Policy, and Economic In- equality in Twenty-One Countries. London.

Rhodes, M. (Hrsg.) (1997): Southern European Welfare States: Between Crisis and Reform, London.

Sachße, C./Tennstedt, F. (1988): Geschichte der Armenfürsorge in Deutschland. Bd.2, Fürsorge und Wohlfahrtspflege 1871 bis 1929 , Stuttgart. $(\leftarrow$ S. 430)

Schwander, H./Manow, P. (2017): ,Modernize and Die‘? German Social Democracy and the electoral 
Consequences of the Agenda 2010, in: Socio-Economic Review 15(1), S. 117 - 134.

- (2018): Explaining the electoral success of the German right-wing populist AfD, CIS working paper (Center for Comparative and International Studies, University of Zurich), No. 94.

Shalev, M. (2007): Limits and Alternatives to Multiple Regression in Comparative Research, in: Mjoeset, L./Clausen, T. H. (Hrsg.), Capitalisms Compared, Amsterdam, S. 261 - 308.

Siaroff, A. (1994): Work, Welfare and Gender Equality: A New Typology, in: Sainsbury, D. (Hrsg.), Gendering Welfare States, London, S. $82-100$.

- (1999): Corporatism in 24 industrial democracies: Meaning and measurement, in: European Journal of Political Research 38.2, S. 175 - 205.

Troeltsch, E. (1923): Die Soziallehren der christlichen Kirchen und Gruppen. Tübingen.

Watson, S. (2015): The Left Divided: The Development and Transformation of Advanced Welfare State, Oxford.

Wren, A. et al. (2013): The Trilemma Revisited: Institutions, Inequality, and Employment Creation in an Era of ICT-Intensive Service Expansion, in: Wren, A. (Hrsg.), The Political Economy of the Service Transition, Oxford, S. $108-146$.

Wren, A./Iversen, T. (1998): Equality, Employment, and Budgetary Restraint: The Trilemma of the Service Economy, in: World Politics 50,4, S. $507-546 .(\leftarrow$ S. 431) 